\title{
Data Throughput Assessment of 4T4R MIMO Technique on LTE Wireless Cellular Networks
}

\author{
Joseph M. Mom \\ Department of Electrical and \\ Electronics Engineering, Federal \\ University of Agriculture, Makurdi
}

\author{
George K. Azever \\ Department of Electrical and \\ Electronics Engineering, Federal \\ University of Agriculture, Makurdi
}

\author{
Moses S. Maor \\ Department of Electrical and \\ Electronics Engineering, Federal \\ University of Agriculture, Makurdi
}

\begin{abstract}
Long Term Evolution (LTE) known as 4G technology is developed to solve exponential demand for higher data rate by the users. This research work carried out measurement on a captured LTE wireless network of a 4- way Transmit, 4-way Receive (4T4R) multiple-input multiple-output (MIMO) optimization technique and existing $2 \times 2$ MIMO to assess the throughput. The assessment was conducted on a mobile network set up of four sites and unit inter connection on LTE continuous coverage with inter-site distance of $500 \mathrm{~m}$ using two terminals user equipment to download and upload data by drive test. Monitoring and results collection were carried out using Local monitoring Terminal (LMT) and Test mobile system (TEMs) Drive Test (DT) kit as well as analysis of data using TEMs DT kit and NetPerSec. The results showed that Terminal 1 recorded a maximum achievable downlink and uplink throughput of 47.5 Mbps and 14.2 Mbps respectively against the system baseline $2 \times 2$ MIMO of $33.6 \mathrm{Mbps}$ and $10.7 \mathrm{Mbps}$ respectively. Terminal2, on the other hand, achieved maximum downlink and uplink throughput of 44.0 Mbps and 14.1 Mbps respectively higher than the same baseline $2 \times 2$ MIMO. This improvement indicates that increasing the number of transmit and receive antennas expands the network capacity thereby yielding more throughput.
\end{abstract}

\section{General Terms}

LTE, Cellular Network, optimization technique, drive test.

\section{Keywords}

Throughput, Downlink, Uplink, MIMO, data rate.

\section{INTRODUCTION}

Long Term Evolution (LTE) is the evolution of the ThirdGeneration $(3 \mathrm{G})$ of mobile communications that create a new radio-access technology which provide high data rates, low latency and high spectral efficiency [1]. Due to the new technologies that have inherent capacity for higher data rate consumption, there is great need to optimize with basic features techniques. Since, network conditions and User Equipment (UE) capabilities differ greatly, a high degree of flexibility is required in order to have maximum throughput. One of such solutions is the improvement of performance and spectral efficiency using MIMO technology, which is one of the main features of the LTE develop to improve data throughput and spectral efficiency above that obtained by the use of OFDM [2]. Although MIMO adds complexity to the system in terms of processing and the number of antennas required, it enables far high data rates to be achieved along with much improved spectral efficiency [3]. As a result, MIMO has been included as an integral part of LTE to enhances space dimension, network capacity, increase range and improve reliability without additional bandwidth as well as increased transmit power [2], [4].

The aim of this study is to assess the impact of a $4 \times 4$ MIMO optimization technique on an existing $2 \times 2$ MIMO optimization technique on LTE network in term of data rate throughput.

\section{Mathematical formulation of $4 \times 4$ MIMO}

The equations and parameters that are relevant for the mathematical computation of peak data rate using MIMO technologies of wireless cellular networks are discussed as follows:

\subsection{MIMO Channel Capacity}

Wireless cellular networks with Single-Input-Single-Output (SISO) systems offer limited channel capacity. The capacity of such systems is given by Shannon capacity theorem in a mathematical form [5] as

$C=B \times \log _{2}(1+S / R) b i t / s$

Where $\mathrm{C}=$ capacity, $\mathrm{B}=$ bandwidth of the systems, $\mathrm{S} / \mathrm{R}=$ signal to noise ratio.

Therefore, MIMO systems are the one with multiple antennas at transmitting end and multiple antennas at receiving end. By using MIMO, these additional paths can be used to advantage to provide additional robustness to the radio link by improving the signal to noise ratio, or by increasing the link data capacity [2] [3].

The capacity of the MIMO systems for $n$ transmitters and $n$ receivers deriving from equation 1 are given by the relation

$\mathrm{C}=\mathrm{B} \times \log _{2}(1+\mathrm{nT} \times \mathrm{nR} \times \mathrm{S} / \mathrm{R}) \mathrm{bit} / \mathrm{s}$

Where, $\mathrm{nT}=$ number of transmitter antenna, $\mathrm{nR}=$ number of receiver antenna.

But, by applying space-time coding techniques where the signal is coded. The capacity of the MIMO systems is given $C=\min (n T, n R) \times B \times \log _{2}(1+S / R) b i t / s$

Where, $\operatorname{Min}(\mathrm{nT}, \mathrm{nR})=$ minimum of $\mathrm{nT}$ and $\mathrm{nR}$

For the purpose of this work, $4 \times 4$ MIMO is used where $n T=4$ and $\mathrm{nR}=4$.

\subsection{MIMO Channel Model}

In MIMO systems, the transmitter sends multiple streams of signals by multiple transmit antennas. As described in the work of [2], the transmit streams of signals go through a matrix channel which consists of all $\mathrm{N}$ transmit and $\mathrm{N}$ received paths between the $\mathrm{Nt}$ transmitting antennas and $\mathrm{Nr}$ receiving antennas. The receiving multiple antennas on getting the received signal vectors decodes them into the original information. A narrowband flat fading MIMO system 
is thus modeled with Equation (4) below. The variables are matrices of complex numbers rather than scalar numbers.

$$
\mathbf{r}=\mathbf{H s}+\mathbf{n}
$$

Where $\mathrm{r}$ and $\mathrm{s}$ are the received and transmit vectors, while $\mathrm{H}$ and $\mathrm{n}$ are the channel matrix.

\section{METHODOLOGY}

Four mobile network sites labelled A, B, C and D of a service provider were selected as shown in Appendix A (Figure 1). The test trial cluster was chosen based on LTE continuous coverage with inter-site distance of about $500 \mathrm{~m}$ to reduce the impact on Multi path effect in the said cluster.

The Antenna setup of one-box 4-Port and radio Unit inter connection of same length Jumper Cables with an Engineering tolerance of $\leq 1 \mathrm{~m} \& 1 \mathrm{Db}$ is shown in Figure 2. Two terminals, Terminal 1 and Terminal 2 which are User Equipment (UEs) were used to download and upload data. The choice of the terminals was based on, Terminal 1 (Sony Z5 Ultra (QualcommSnapdragon 820)) is the World $1^{\text {st }} 4 \times 4$ MIMO Phone [6] supported by a $4 \times 4$ MIMO. Whereas Terminal 2 (Huawei B618) with features Such as 4G LTE speeds up to $600 \mathrm{Mbps}$, WiFi for 64 devices at a time, Wireless-AC WiFi speeds up to $1300 \mathrm{Mbps}, 2 \mathrm{LAN}$ ports (including $1 \mathrm{WAN} /$ LAN), 1 RJ11 port for phone, printer or fax, 1 Micro SIM card slot (Dual-Free), 2 External Aerial Ports (TS-9), 1 USB 2.0 Port, Huawei HiLink App and Weight of 700 grams [7].

The similarity and difference in the behavior of the terminals were checked under controlled radio conditions. The behavior of the data streams was monitored using network Local monitoring Terminal (LMT). The results were collected using TEMs drive test Kit and analyzed via DT tool and NetPerSec, a universal internet tool for data speed test [8].

For the purpose of validation, the same methodology was adopted for existing $2 \times 2$ MIMO with test UE (Huawei E5375). HUAWEI E5375 Hotspot is an LTE Cat4 and WiFi $2 \times 2$ technology with maximum download speeds of up to $150 \mathrm{Mbps}$ [9].

Network Characteristics of the trial cluster on which study was conducted are depicted in Table 1.

Table 1: Network Characteristics of the trial cluster

\begin{tabular}{|c|c|c|}
\hline $\mathrm{S} / \mathrm{N}$ & Parameters & Setting/Description \\
\hline 1 & Spectrum & $\begin{array}{c}\text { Refarm technology on L1800 } \\
\text { band } 3\end{array}$ \\
\hline 2 & Bandwidth & $5 \mathrm{MHz}$ \\
\hline 3 & $\begin{array}{l}\text { Number of } \\
\text { OFDMA } \\
\text { subcarriers }\end{array}$ & 25 \\
\hline 4 & $\begin{array}{l}\text { Modulation } \\
\text { Technique }\end{array}$ & 64QAM \\
\hline 5 & Diversity & $\begin{aligned} \mathrm{DL}= & 2 \times 2 \mathrm{MIMO}, 4 \times 4 \mathrm{MIMO} \\
& \& \mathrm{UL}=1 \mathrm{X} 2 \mathrm{MIMO}\end{aligned}$ \\
\hline 6 & Duplex Mode & FDD \\
\hline 7 & OFDMA Symbol & Normal Symbol \\
\hline 8 & TTI & $1 \mathrm{~ms}$ \\
\hline
\end{tabular}

The maximum achievable DL/UP throughput, receive diversity and radio parameters results captured on TEMs drive test kit for $2 \times 2$ MIMO and $4 \times 4$ MIMO are shown in the Appendices B, C,D and E respectively and the discussion of results are presented section 5 .

\section{RESULTS AND DISCUSSIONS}

The discussions are centered on the drive test on $2 \times 2$ MIMO used as baseline and $4 \times 4$ MIMO of the two Terminals 1 and 2achievable data throughput results obtained. The results of captured throughput results, 4 streams of data during the test for verification (4-way receive diversity) and radio parameters captured during the study are shown in the appendix. The assessment of the study was done to certain the impact of MIMO technology on data rate (throughput) improvement in LTE deployment.

\subsection{MIMO Throughput Results}

The maximum achievable downlink and uplink throughput results evaluated for 2x2 MIMO with Huawei E5375 Hotspot and $4 \times 4$ MIMO for Terminal 1 and 2 as UEs are extracted from Appendices B, C and D were computed as presented in Table 2.

Table 2: Results obtained for Analysis

\begin{tabular}{|c|c|c|c|c|}
\hline S/N & Test UE & $\begin{array}{c}\text { MIMO } \\
\text { used }\end{array}$ & $\begin{array}{c}\text { Max.DL } \\
\text { throughput } \\
\text { (Mbps) }\end{array}$ & $\begin{array}{c}\text { Max.UL } \\
\text { throughput } \\
\text { (Mbps) }\end{array}$ \\
\hline 1 & $\begin{array}{c}\text { Huawei } \\
\text { E5375 }\end{array}$ & $\begin{array}{c}2 \times 2 \\
\text { MIMO }\end{array}$ & 33.6 & 10.7 \\
\hline 2 & Terminal 1 & $\begin{array}{c}4 \times 4 \\
\text { MIMO }\end{array}$ & 47.5 & 14.2 \\
\hline 3 & Terminal 2 & $\begin{array}{c}4 \times 4 \\
\text { MIMO }\end{array}$ & 44.0 & 14.1 \\
\hline
\end{tabular}

\subsubsection{Results of Terminal 1Throughput Obtained}

From the results of Table 2 for Terminal 1, maximum downlink throughput achieved was $47.5 \mathrm{Mbps}$. This represented about $29.3 \%$ increase from the baseline $33.6 \mathrm{Mbps}$ of $2 \times 2$ MIMO techniques used on the Network. Whereas maximum uplink throughput achieved was $14.2 \mathrm{Mbps}$. This represented about $32.7 \%$ increase from the existing $10.7 \mathrm{Mbps}$ of 2x2 MIMO technique used on the Network. This indicates that increasing the number of transmitters and receivers of antennas enhance the network capacity thereby increase data throughput of the network.

\subsubsection{Results of Terminal 2 Throughput Obtained}

In Table 2 for Terminal 2, Maximum downlink throughput achieved was $44.0 \mathrm{Mbps}$. This represented about $23.7 \%$ increase from the existing $33.6 \mathrm{Mbps}$ of $2 \times 2 \mathrm{MIMO}$ technique used on the Network, while maximum uplink throughput achieved was $14.1 \mathrm{Mbps}$. This represented $24.1 \%$ increase from the baseline $10.7 \mathrm{Mbps}$ of $2 \times 2 \mathrm{MIMO}$ techniques used on the Network. This percentage increase shows that $4 \times 4$ MIMO is more optimized to improve capacity as compared to baseline 2x2 MIMO technique. 


\subsubsection{Results of Radio Parameters}

Figure 8 in Appendix D, shows excellent radio conditions and parameters during the study as defined in [10]. It is observed that RSRP and RSRQ were maintained at a mean value of $68.00 \mathrm{dBm}$ and $-13.00 \mathrm{dBm}$ respectively. This represents a very good radio condition of the captured network. Maximum RANK Indicator was also achieved (RANK 3). Ranking is done from 0 to 3, hence RANK 3 implies that all the four data streams were used in the study.

\section{CONCLUSION AND RECOMMENDATIONS}

The research work has established great improvement in user throughput using two capable UEs on a $4 \times 4$ MIMO technique as compared to the existing $2 \times 2 \mathrm{MIMO}$ technique.

By using this technique, Network Operators would:

1. Improve Max UL throughput by $32.7 \%$ (10.6 Mbps to $14.2 \mathrm{Mbps}$ ) and Max DL throughput by $29.3 \%$ (33.6 Mbps to 47.5 Mbps) respectively using Terminal 1.

2. Improve MaxUL throughput by $23.7 \%$ (10.6Mbps to $14.1 \mathrm{Mbps}$ ) and Max DL throughput by $24.1 \%$ (33.6 Mbps to $44 \mathrm{Mbps}$ ) using Terminal 2

Hence, this improvement has demonstrated the efficiency of the technique through drive test measurement. This technique would absolutely increase user speed thereby enhancing system capacity and improving average quality of cellular network system.

It is therefore recommended that cellular network service providers should adopt this strategy in enhancing data speed using LTE so as to improve and enhance subscriber experience.

\section{ACKNOWLEDGMENTS}

The Authors thank all those who have contributed towards success of this research work.

\section{REFERENCES}

[1] Tshiteva, D .and Anthony,L. 2011.Downlink Scheduling in 3GPP Long Term Evolution (LTE). Wireless and mobile Communication (WMC)Group Faculty of Electrical Engineering, Mathematics and Computer Science, Delft University of Technology.

[2] Olabode, B. I., Augustus, E. I., Atayero, A.A. 2017.MIMO Optimization Techniques and Their Application in Maximizing Throughput for3GPP HSPA+.Scientific \& Academic Publishing, Electrical\& Information Engineering Dept Covenant University, Ota, 'Nigeria.

[3] Evans, B. G., and Baughan, K., 2002. Visions of 4G," Electronics and Communication Engineering Journal.12(6): pp 293-303.

[4] Praveen, V. and Sven, N. 2016. Maximizing LTE performance with MIMO systems. Master Thesis in Electrical Engineering,Linnaeus University Sweden.

[5] Kritika, S., Nishu, R., Ankita, S., Dolly, S., Seema, V., Tanya, S. 2014. Study and Capacity Evaluation of SISO, MISO and MIMO RF Wireless Communication Systems. International Journal of Engineering Trends and Technology (IJETT) - 9(9); pp 436-440.

[6] Mobile World Congress (MWC). 2016. World 1st 4x4 MIMO Phone, Sony Z5 Ultra (NGTUE). (QualcommSnapdragon 820).

[7] https://www.4gltemall.com/blog/tag/huawei-b618review/. Released on $19^{\text {th }}$ March, 2018.

[8] https://www.academia,edu/675747/LTE_Drive_Test_Pro cedure. Downloaded on $10^{\text {th }}$ January, 2019.

[9] https://www.4gltemall.com/blog/tag/huawei-e5375/. Released on $16^{\text {th }}$ July, 2013.

[10] Harish, M. 2016. LTE Drive Test Parameters. Post Graduate Diploma in Management at Great Lakes Institute of Management. http//www.lte drives test parameters.com.

\section{APPENDIX A}

Site selection and Antenna \& Radio Unit layout for the Study

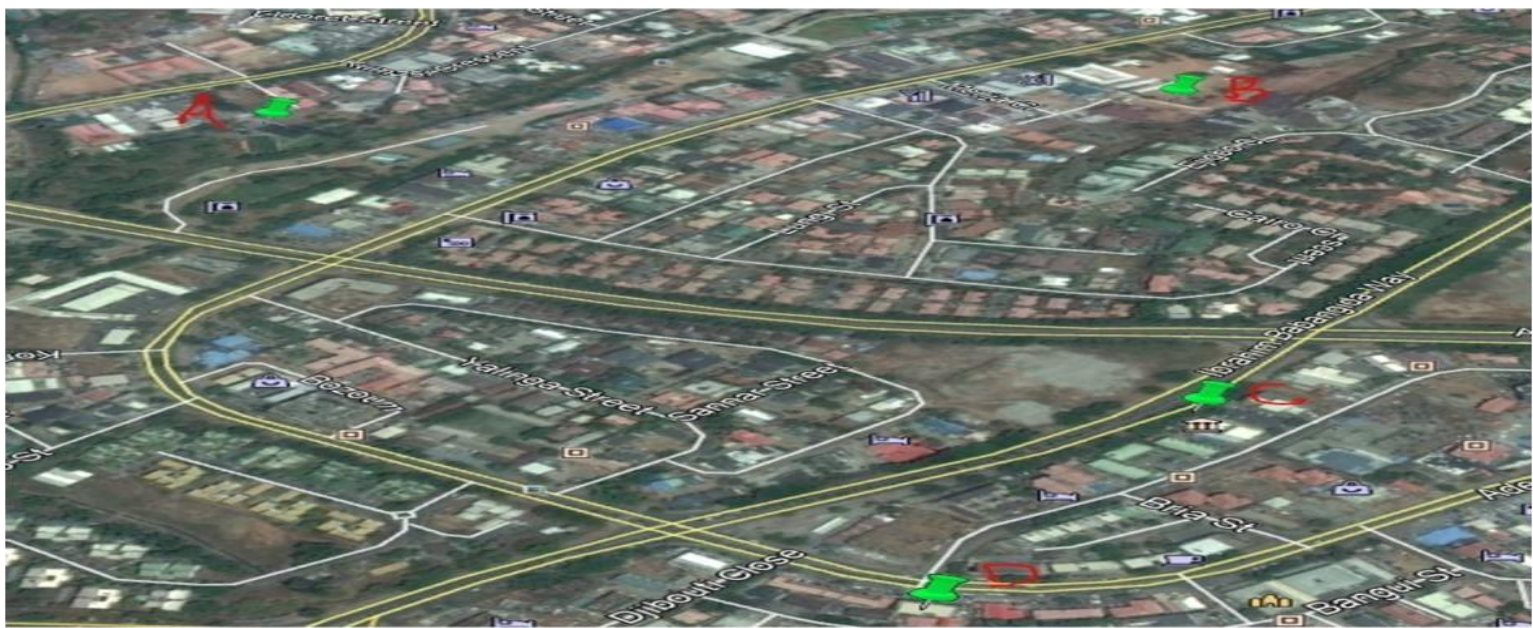

Figure 1: Site captured for the Study 


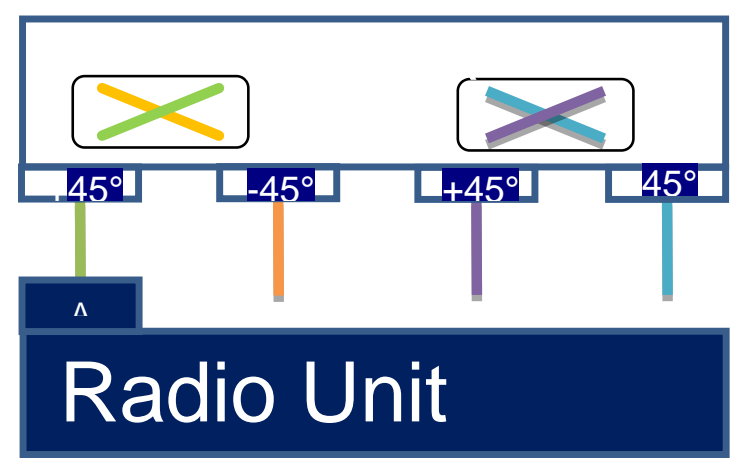

Figure 2: Antenna and Radio Unit layout

\section{APPENDIX B}

2X2 MIMO

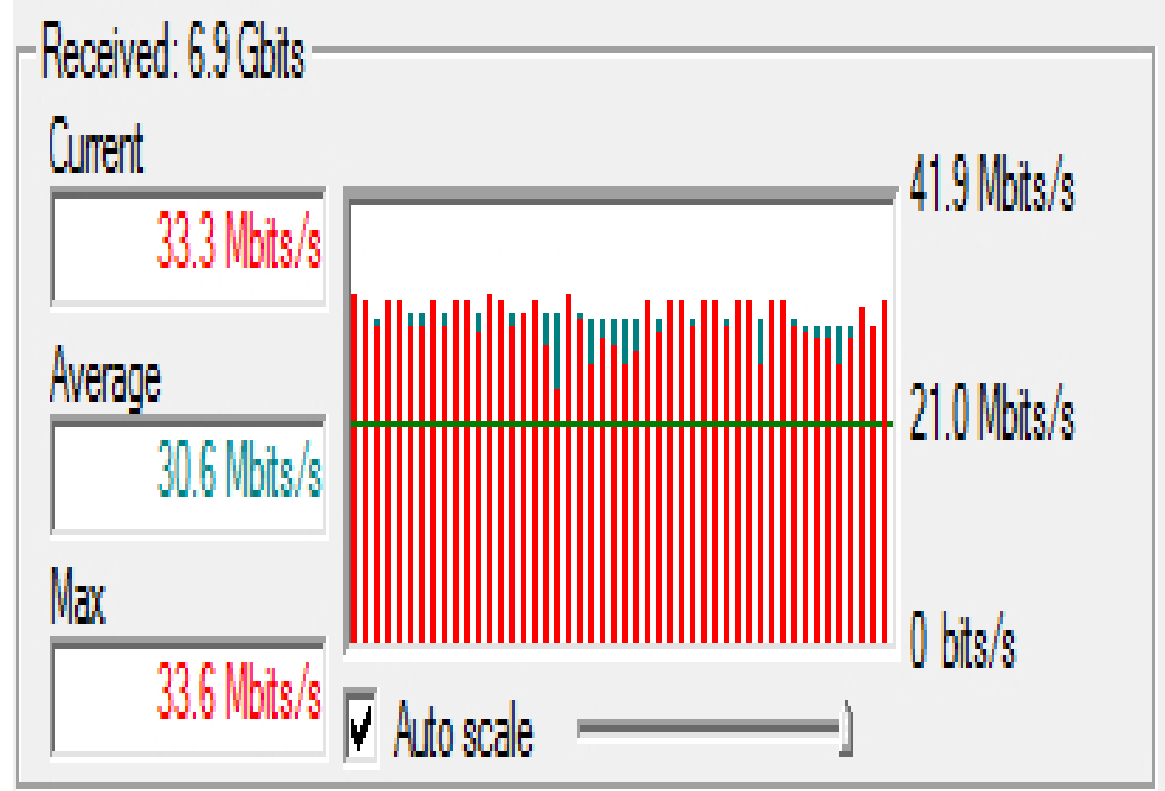

-

Figure 3: Captured Downlink Throughput results of 2x2 MIMO

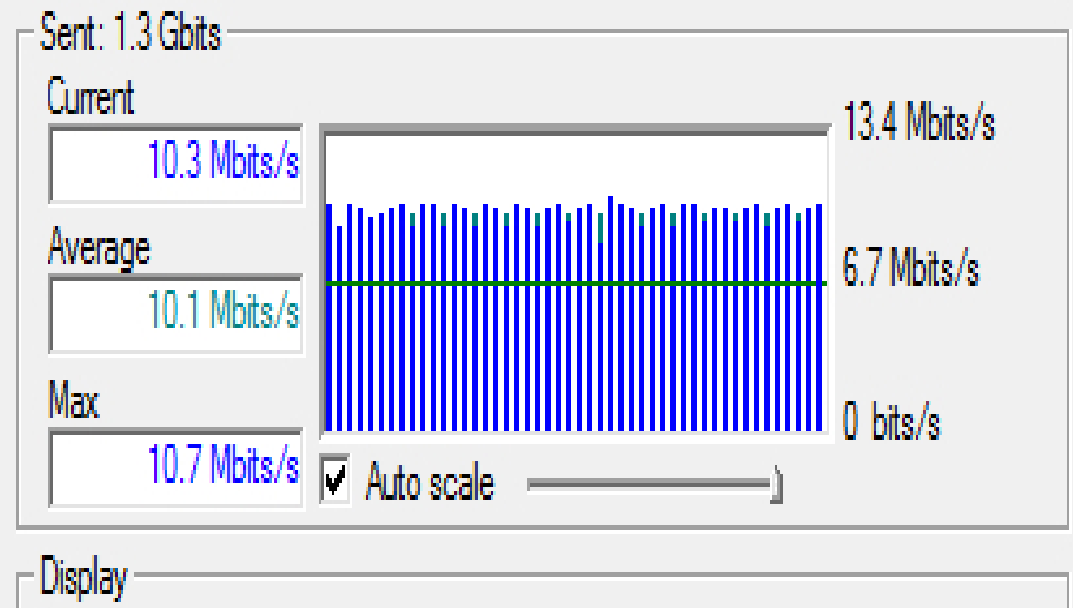

Figure 4: Captured Uplink Throughput results of $2 \times 2$ MIMO 


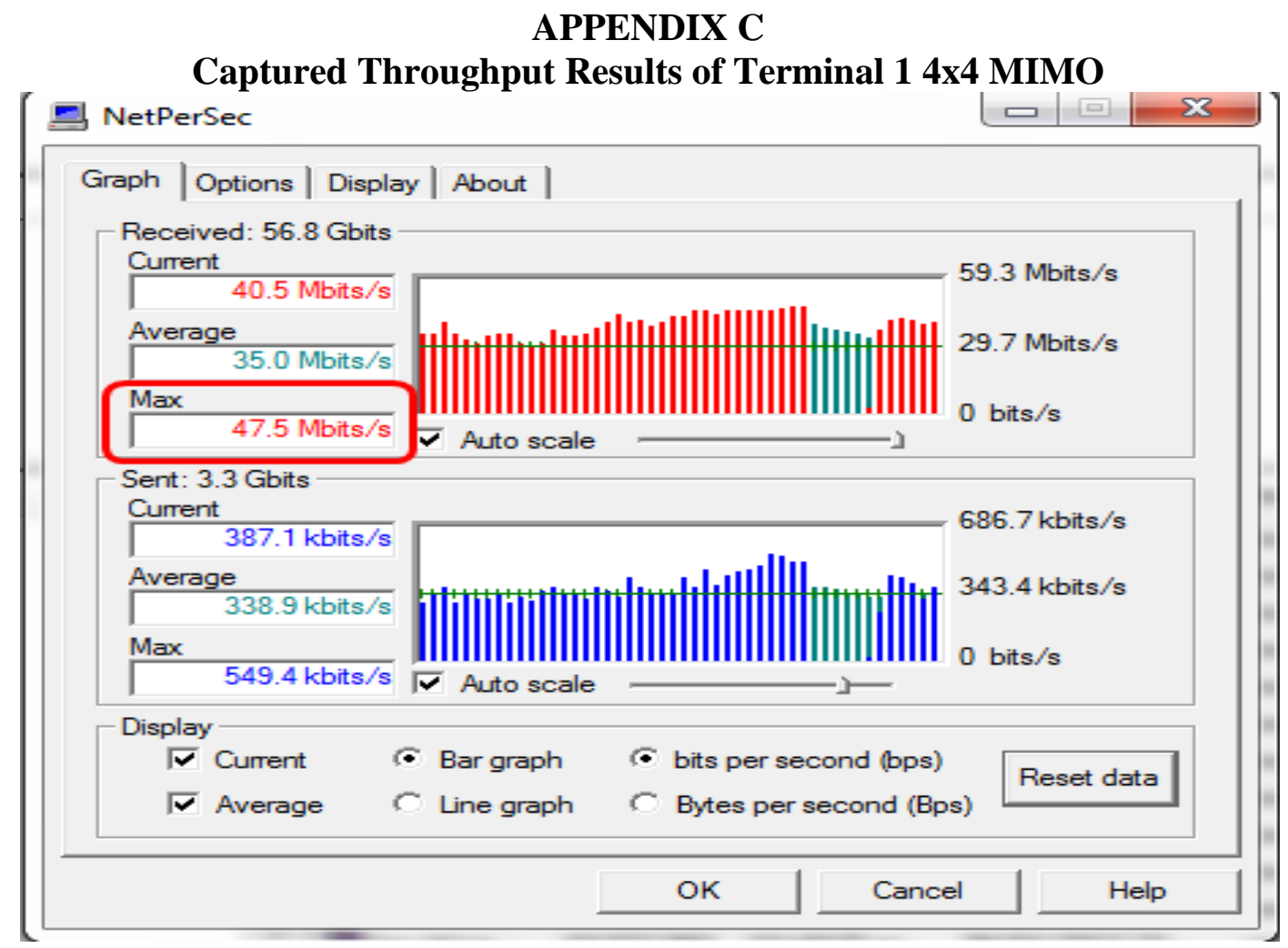

Figure 5: Captured Downlink Throughput Results of 4x4 MIMO

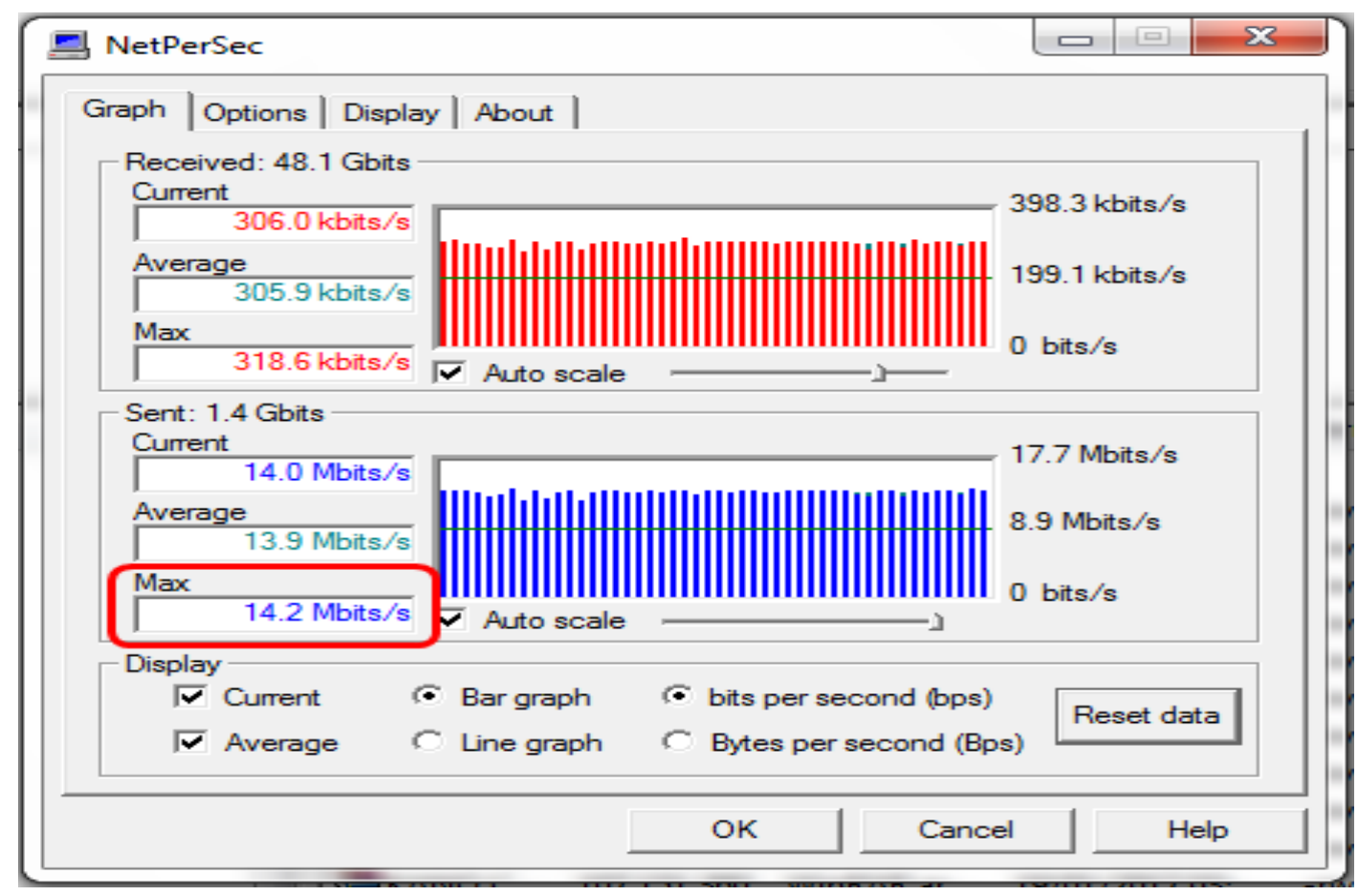

Figure 6: Captured Uplink Throughput results 4x4 MIMO 
APPENDIX D

Captured Throughput Results of Terminal 2, 4x4 MIMO

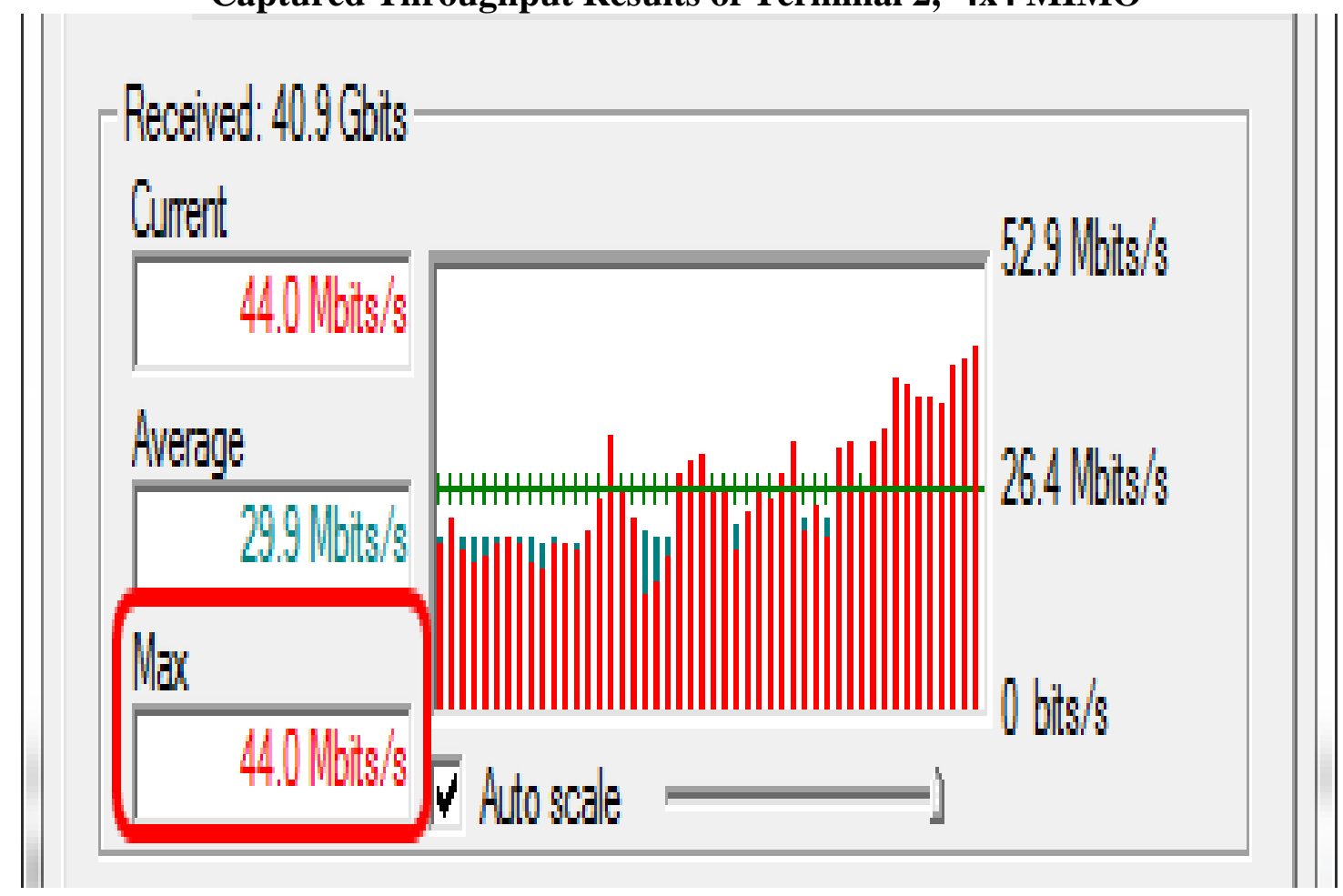

Figure 7: Captured Downlink Throughput results

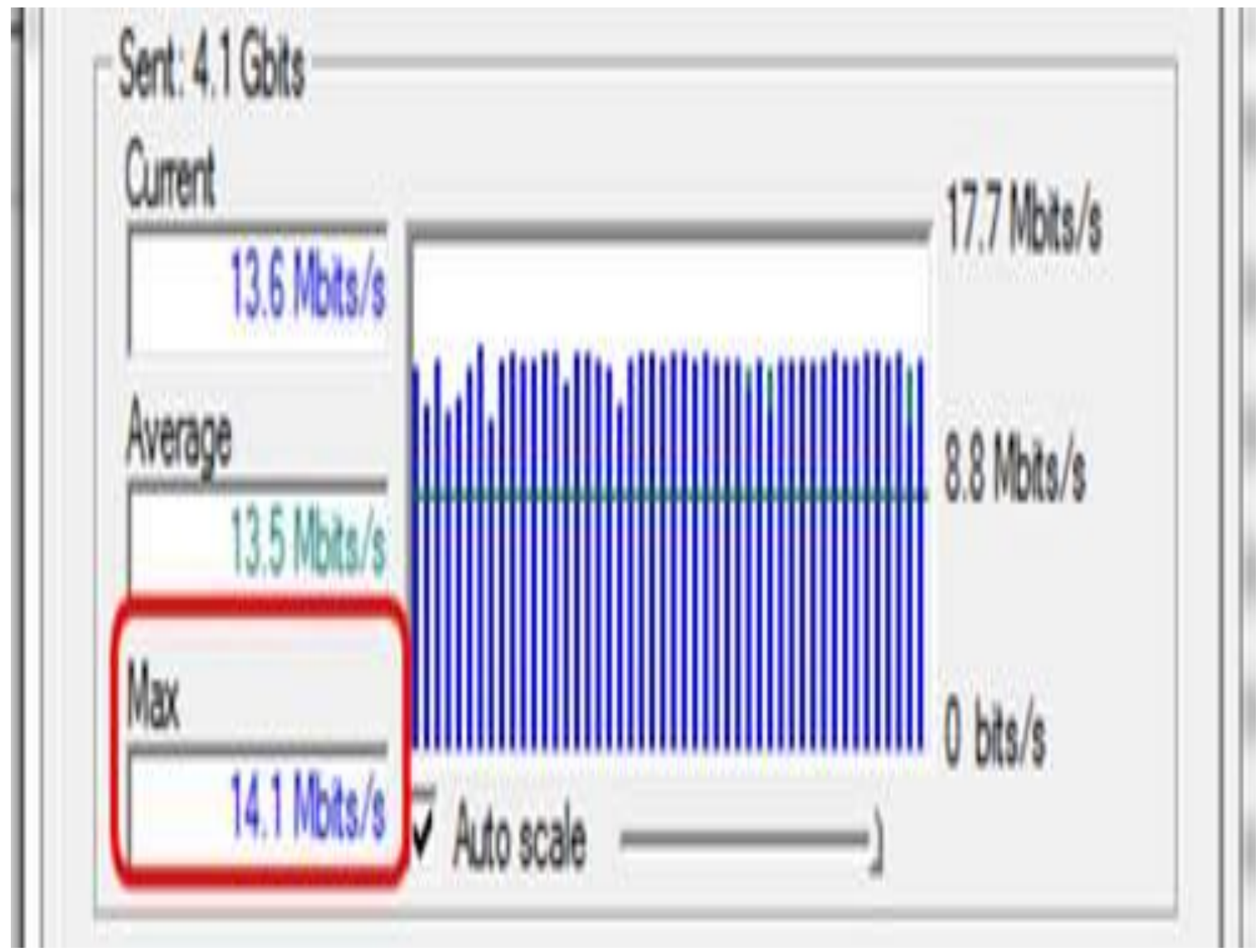

Figure 8: Captured Uplink Throughput results 
APPENDIX E: Radio Parameters Captured

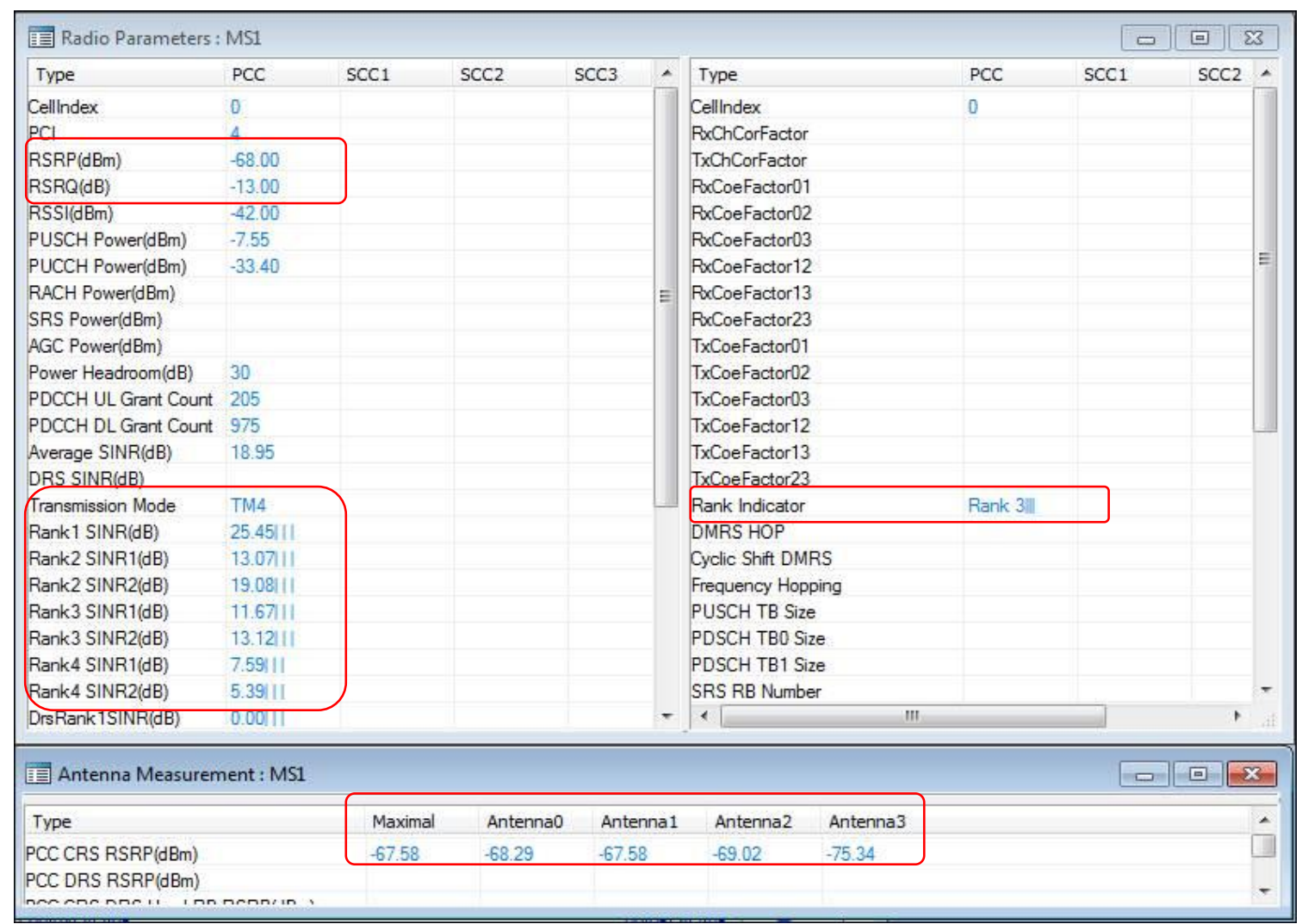

\begin{tabular}{|c|c|c|c|c|c|c|c|c|c|c|c|c|}
\hline \multicolumn{6}{|c|}{ 国 Radio Parameters : MSI } & \multirow{2}{*}{\multicolumn{2}{|c|}{ Type }} & & \multirow{2}{*}{\multicolumn{2}{|c|}{$\frac{\square}{\operatorname{scc} 1}$}} & \multirow{2}{*}{$\frac{\text { (a) }}{\operatorname{SCC} 2}$} & \multirow{2}{*}{$\frac{83}{14}$} \\
\hline Type & PCC & $\operatorname{SCC} 1$ & $\operatorname{SCC} 2$ & $\mathrm{SCC} 3$ & $\wedge$ & & & PCC & & & & \\
\hline Cellindex & 0 & & & & & \multicolumn{2}{|l|}{ Cellindex } & 0 & & & & \\
\hline $\mathrm{PCl}$ & 4 & & & & & \multicolumn{2}{|l|}{ RxChCorFactor } & & & & & \\
\hline $\operatorname{RSRP}(\mathrm{dBm})$ & -68.00 & & & & & \multicolumn{2}{|l|}{ TxChCorFactor } & & & & & \\
\hline $\operatorname{RSRQ}(\mathrm{dB})$ & -13.00 & & & & & \multicolumn{2}{|l|}{ RxCoeFactor 01} & & & & & \\
\hline $\operatorname{RSSI}(\mathrm{dBm})$ & -42.00 & & & & & \multicolumn{2}{|l|}{$\mathrm{R} \times \mathrm{C}$ CoeFactor 02} & & & & & \\
\hline PUSCH Power(dBm) & -7.55 & & & & & \multicolumn{2}{|l|}{ RxCoeFactor 03} & & & & & 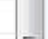 \\
\hline PUCCH Power(dBm) & -33.40 & & & & & \multicolumn{2}{|l|}{ Rxcoefactor 12} & & & & & $\equiv$ \\
\hline RACH Power(dBm) & & & & & $\equiv$ & \multicolumn{2}{|l|}{ RxCoeFactor 13} & & & & & \\
\hline SRS Power(dBm) & & & & & & \multicolumn{2}{|l|}{ RxxoeFactor 23} & & & & & \\
\hline AGC Power(dBm) & & & & & & \multicolumn{2}{|l|}{ TxCoeFactor01 } & & & & & \\
\hline Power Headroom(dB) & 30 & & & & & \multicolumn{2}{|l|}{ TxCoeFactor 02} & & & & & \\
\hline PDCCH UL Grant Count & 205 & & & & & \multicolumn{2}{|l|}{ TxCoeFactor 03} & & & & & \\
\hline PDCCH DL Grant Count & 975 & & & & & \multicolumn{2}{|l|}{ TxCoeFactor 12} & & & & & $\square$ \\
\hline Average SINR(dB) & 18.95 & & & & & \multicolumn{2}{|l|}{ TxCoeFactor 13} & & & & & \\
\hline DRS SINR(dB) & & & & & & \multicolumn{2}{|l|}{ IxCoefactor 23} & & & & & \\
\hline Transmission Mode & TM4 & & & & L & \multicolumn{2}{|l|}{ Rank Indicator } & Rank 3ill & & & & \\
\hline Rank1 SINR(dB) & 25.45111 & & & & & \multicolumn{2}{|l|}{ DMRS HOP } & & & & & \\
\hline Rank2 SINR1(dB) & 13.07111 & & & & & \multicolumn{2}{|c|}{ Cyclic Shift DMRS } & & & & & \\
\hline Rank2 SINR2(dB) & 19.08111 & & & & & \multicolumn{2}{|c|}{ Frequency Hopping } & & & & & \\
\hline Rank3 SINR1(dB) & 11.67111 & & & & & \multicolumn{2}{|c|}{ PUSCH TB Size } & & & & & \\
\hline Rank3 SINR2(dB) & $13.12 \| 11$ & & & & & \multicolumn{2}{|c|}{ PDSCH TBO Size } & & & & & \\
\hline Rank4 SINR1(dB) & 7.59111 & & & & & \multicolumn{2}{|c|}{ PDSCH TB1 Size } & & & & & \\
\hline Rank4 SINR2(dB) & 5.39111 & & & & & \multicolumn{2}{|c|}{ SRS RB Number } & & & & & $r$ \\
\hline DrsRank1SINR(dB) & 0.0011 & & & & + & $1 \square$ & III & 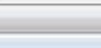 & & & 1 & aif \\
\hline 国 Antenna Measurem & lent : MS1 & & & & & & & & & $\square$ & 回 5 & $\mathrm{x}$ \\
\hline Type & & Maximal & Antenna0 & Ante & Ina1 & Antenna 2 & Antenna3 & & & & & $\hat{A}$ \\
\hline PCC CRS RSRP(dBm) & & -67.58 & -68.29 & -67.5 & & -69.02 & -75.34 & & & & & Q \\
\hline PCC DRS RSRP(dBm) & mannim & & & & & & & & & & & - \\
\hline
\end{tabular}

Figure 8: Radio Parameters Captured during the Study 